\title{
Novel Convenient Method to Determine Wettability and Dispersibility of Dairy Powders
}

\author{
Jeae Lee, Changhoon Chai, Dong June Park ${ }^{1}$, Kwangsei Lim²*, and Jee-Young Imm* \\ Department of Foods and Nutrition, Kookmin University, Seoul 136-702, Korea \\ ${ }^{1}$ Korea Food Research Institute, Seongnam 463-746, Korea \\ ${ }^{2} R \& D$ Center, Maeil Dairies, Co. Ltd., Pyungtaek 451-861, Korea
}

\begin{abstract}
This study was carried out to develop a simple, convenient, and reproducible testing device to determine wettability and dispersibility of dairy powders. The testing device consists of a sieve $(150 \mu \mathrm{m})$ attached to a sample chamber, sensors mounted on a supporting body and a main control unit containing a display panel. The sensors detect the difference in electrical resistance between air and water. A timer is automatically triggered by the sensor when the bottom of sample-loaded chamber contacts water in the petri dish. Wettability and dispersibility of commercial skim milk powders (SMPs) produced at different heating strengths (low-, medium-, and high-heat SMP) are compared using the new testing device. Wettability of the SMPs were correlated with particle size and are found to increase in the order of medium-, low-, and high-heat SMP regardless of the amount of sample tested. Dispersibility of SMPs showed the same trend and high heat-SMP which has the smallest particle size resulted in the lowest dispersibility. Unlike existing methods, the new testing device can determine both wettability and dispersibility of powders and successfully detected differences among the samples.
\end{abstract}

Keywords: wetting, dispersibility, skim milk powder, heating strength, testing device

\section{Introduction}

Powder is one of the most convenient forms to transport and store food components, and there is an increase in the industrial applications of functional food powders. Dairy-based powders have been widely used in the food industry because of their good nutritional quality and mild flavor. In order to achieve maximum functionality powders should be readily dispersible in a medium and remain dissolved or suspended over time regardless of environmental conditions. There have been many attempts to improve the hydration properties of dairy powders by altering heating regimes or introducing agglomeration processes into their manufacture (Gaiani et al., 2007).

The stages in the reconstitution of powders can generally be categorized into wetting, sinking, dispersing, and complete dissolution although these stages are not clearly

\footnotetext{
*Corresponding authors: Jee-Young Imm, Department of Foods and Nutrition, Kookmin University, Seoul, 136-702, Korea. Tel: +82-2-910-4772, +Fax: 82-2-910-5249, E-mail: jyimm@kookmin.ac.kr; Kwangsei Lim, R\&D Center, Maeil Dairies, Co. Ltd., Pyungtaek 451-861, Korea. Tel: +82-31-612-3902, Fax: +82-31668-0247, E-mail: kwangslim@maeil.com
}

differentiable in real situations (Fang et al., 2008). GEA Niro methods are popularly used in the dairy industry to evaluate several of the reconstitution properties of powders. However, these methods, particularly wettability testing methods (Niro, 2005; Niro, 2006) have some inherent problems in their experimental setup (i.e., samples often stick to the funnel or plate) that can easily result in errors, and they lack reliability and sensitivity.

Several more advanced techniques have been proposed to investigate the dissolution behavior of food powders. Mimouni et al. (2009) determined the size distribution of milk protein concentrate (MPC, $85 \%$ protein) particles during rehydration by using static light scattering. They reported that the low solubility index of MPC85 at room temperature was due to the slow dissolution kinetics. The dynamic dissolution profile of MPC at various temperatures and for varying particle sizes was measured by focused beam reflectance (Fang et al., 2011). In addition, nuclear magnetic resonance (NMR) relaxometry has been used to characterize the rehydration of dairy powders (Davenel et al., 2002).

However, the sophisticated methods require expensive testing equipments and complex analytical tools, and are not convenient for routine use in the dairy industry. The 
objective of this study was to develop a simple, convenient and reproducible testing device to determine the wettability and dispersibility of dairy powders. The principles of the newly developed testing device were explained and the wettability and dispersibility of commercial skim milk powders (SMP) produced by different heat strengths were evaluated by this device.

\section{Material and Methods}

\section{Material}

Low-, medium- and high-heat commercial SMPs were obtained from Lactoland Trockenmilchwerk GmbH (Germany).

\section{Wettability and dispersibility test using the newly developed device}

The testing device consists of a sieve $(150 \mu \mathrm{m})$, a sample chamber (diameter $43 \mathrm{~mm}$, width $90 \mathrm{~mm}$, height 80 $\mathrm{mm}$ ), two sensors mounted upon a supporting body, and the main control unit with a display panel (Fig. 1a). The sieve was attached to the sample chamber (Fig. 1b). A powder sample (SMP, $1 \pm 0.01 \mathrm{~g}$ ) was placed onto the sieve, and the container was then attached to the instrument. Deionized water $\left(35 \mathrm{~mL}, 25^{\circ} \mathrm{C}\right)$ was added to petri dish $(90 \times 20 \mathrm{~mm})$. The sample chamber went down below the water surface by pressing "START" button. A timer is automatically triggered by the sensor when the bottom of sample-loaded chamber contacts water in the petri dish. When sample became totally wet, the button was pressed to stop timer. In order to test dispersibility aliquots of samples $(5 \mathrm{~mL})$ were taken and solid content of the sample was determined by drying samples in a $105^{\circ} \mathrm{C}$ oven for $18 \mathrm{~h}$. The dispersibility of the sample was calculated by following equation:

(solid content in the sample at designated times / solid content in the initial sample) $\times 100$

\section{Particle size analysis}

The samples were dispersed in ethanol and particle size distribution was determined using a laser diffraction particle size analyzer (CILAS 1064 Compagnie Industrielle Des Lasers, France).

\section{Scanning electron microscopy (SEM)}

The size and shape of samples were observed by scanning electron microscopy (SEM). The samples $(0.1 \mathrm{~g})$ were placed on the top of a stub (12 mm diameter) and coated with gold-palladium for $60 \mathrm{~s}$ in an E-1010 ion sputter coater (Hitachi Ltd., Japan). The microstructure of each sample was observed by SEM (model S-2380N; Hitachi Ltd.) and the representative image of each sample was printed.

\section{Statistical analysis}

Wettability and dispersibility analyses were performed in triplicate. The results are expressed as the mean value \pm standard deviation. Statistical analysis was performed using SPSS (SPSS Inc. ver.21, IBM, USA) statistical software. The data were analyzed by one-way analysis of variance (ANOVA), and Duncan's multiple range test was carried out to find significant difference $(p<0.05)$ among the means.

\section{Results and Discussion}

\section{Wettability of skim milk powder determined by newly developed device}

The overall operation procedure of testing device is shown in Fig. 1c. When the "START" button is pressed the sample chamber is lowered to a preset depth of $3 \mathrm{~mm}$ below the surface of water in the petri dish. The two metal sensors are located outside of the sample chamber at the same height as the bottom of chamber (mesh). The metal sensor detects a difference in electrical resistance between air and water, and the timer is triggered when the sample reaches the surface of the water. The penetration depth of sample chamber can be adjusted using a 'UP' or 'DN' button in a control panel, however, the $3 \mathrm{~mm}$ depth is generally close to the height at which a sample floats on the surface of water. If the penetration depth is greater than $10 \mathrm{~mm}$, sample moved backward by the pressure of water that entering through the mesh of sample chamber.

The wettability of commercial SMPs was determined by the newly developed method. As shown in Table 1, wetting time increased in the order of medium-, low-, and high-heat SMP regardless of sample size $(0.5$ and $1 \mathrm{~g})$. Wettability of food powders indicates a molecular interaction between the solid and water phase and is generally considered as the ability of powder particles to overcome the surface tension caused by water (Fang et al., 2008). Initial wetting is commonly the rate-controlling step in the hydration of food powders and rapid wetting is favored by large particles with large pore sizes (Hogekamp and Schubert, 2003). Increased porosity and small contact angle enhance wetting (Gaiani et al., 2005).

Furthermore, it has been reported that free fat contents 


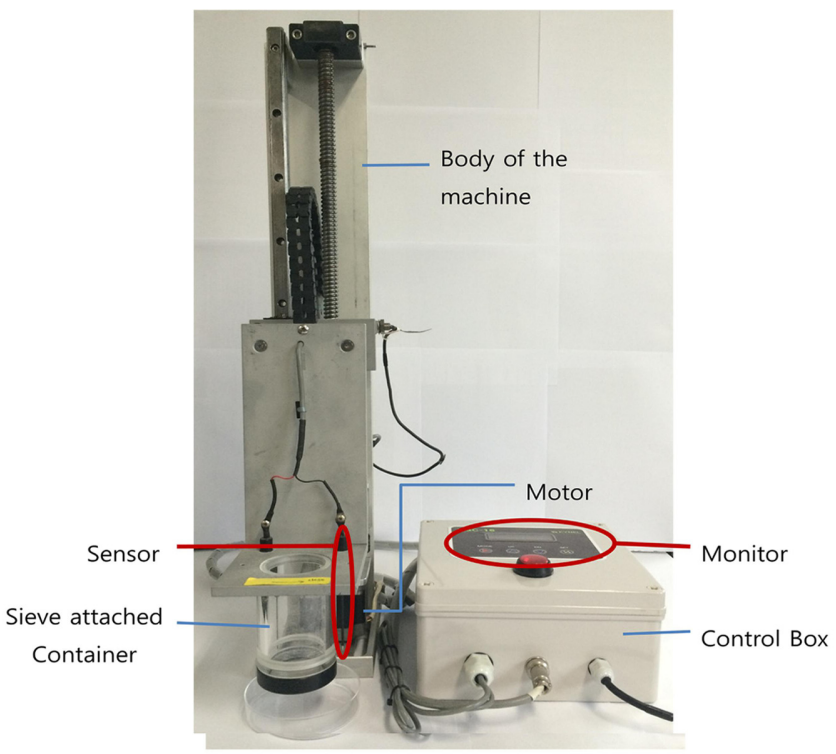

(a)

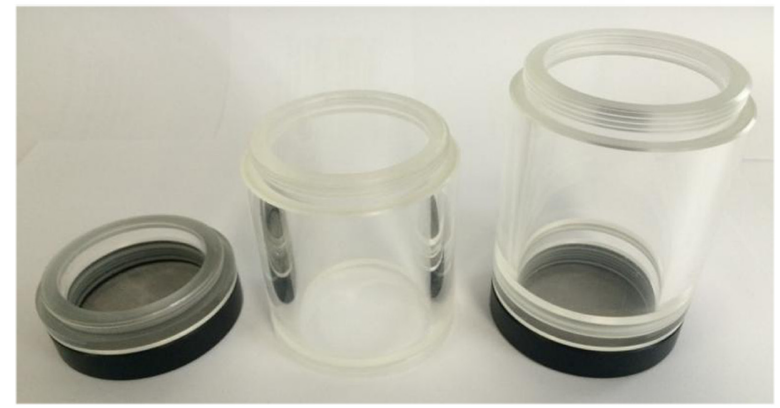

(b)

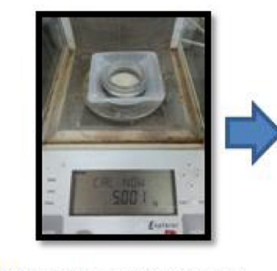

Weigh the sample in sieve attached container
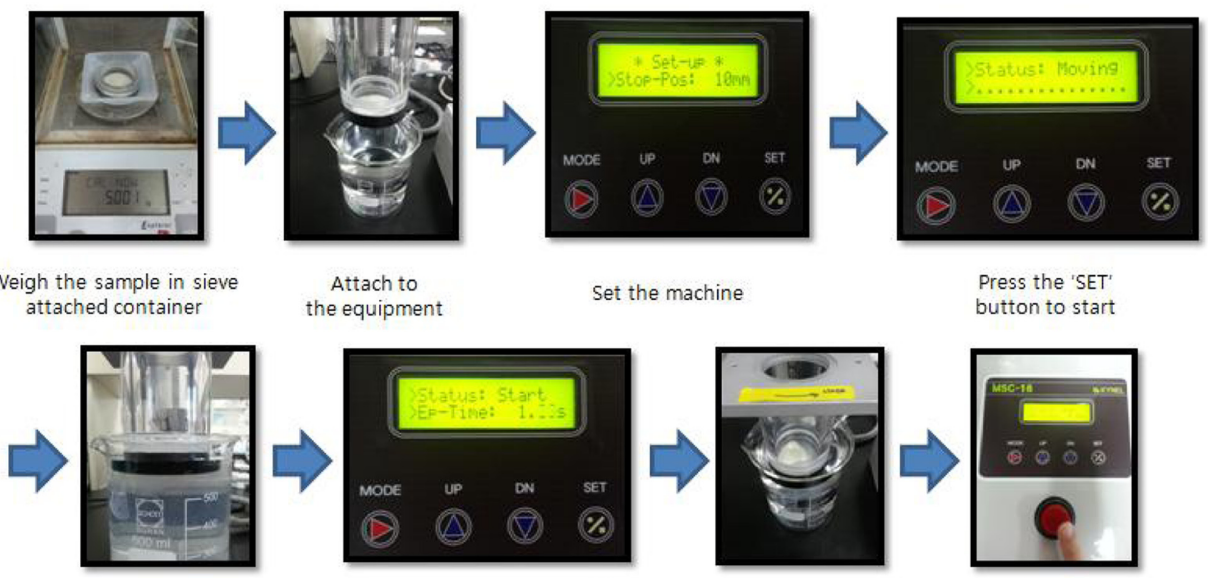
the equipment

Set the machine

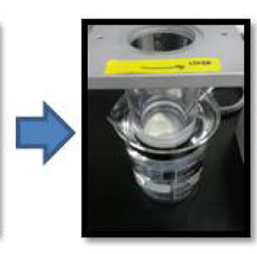

When sample is completely wet
Press the 'SET'

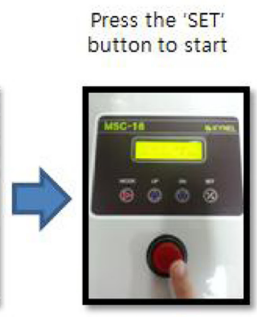

Press the stop button to measure wetting time

(c)

Fig. 1. Structure and operation of the newly developed testing device. (a) Structure of testing device, (b) Assembly of sample chamber unit containing sieve, (c) Operating procedure.

on the powder surface affects wettability by increasing surface hydrophobicity (Hla and Hogekamp, 1999; Kim et al., 2002). In this study, the effect of free fat on SMP wettability should be negligible since free fat content in SMP 
Table 1. Wettability of commercial SMPs with different heating strengths

\begin{tabular}{ccc}
\hline \hline \multirow{2}{*}{ Skim milk powder } & \multicolumn{2}{c}{ Wettability (s) } \\
\cline { 2 - 3 } & $0.5 \mathrm{~g}$ & $1 \mathrm{~g}$ \\
\hline Low heat & $222 \pm 7^{\mathrm{b}}$ & $701 \pm 9^{\mathrm{b}}$ \\
Medium heat & $36 \pm 3^{\mathrm{c}}$ & $195 \pm 3^{\mathrm{c}}$ \\
High heat & $302 \pm 9^{\mathrm{a}}$ & $1,593 \pm 56^{\mathrm{a}}$ \\
\hline
\end{tabular}

Assays were performed in triplicate. Mean $\pm \mathrm{SD}$ values in the same column with different superscripts are significantly different $(p<$ $0.05)$.

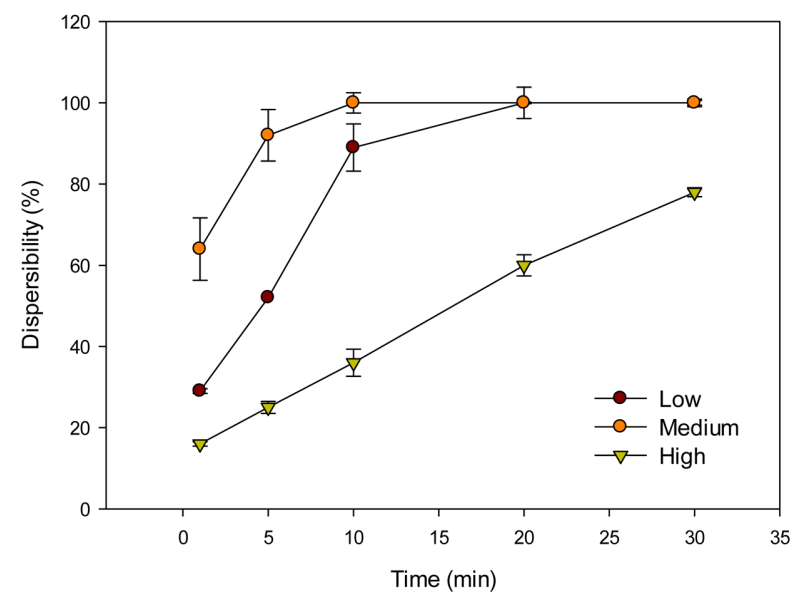

Fig. 2. Dispersibility of commercial SMPs with different heating strengths. Aliquots of samples were taken at the designated time and the solid content of the sample was determined by drying the samples in a $105^{\circ} \mathrm{C}$ oven for 18 h. Dispersibility $(\%)=$ (solid content in the sample at designated times / solid content in the initial sample) $\times 100$.

are not large enough $(1 \mathrm{mg} / \mathrm{g}$ SMP vs. $28 \mathrm{mg} / \mathrm{g}$ whole milk powder) to affect hydrophobicity (Kim et al., 2003).

In the traditional wetting time testing method, powder sample located on the glass plate contacts with water by pulling out the glass plate sideway. The height of the sample piled on the glass plate and stickiness of the samples must be critically controlled to obtain reproducible results. The newly devised testing equipment successfully removed these difficulties which can cause misleading results.

\section{Dispersibility of skim milk powder determined by the newly developed device}

The wetted food particles should ideally disintegrate spontaneously into fine particles. Although the exact definition of dispersibility is not clear it is usually defined as the amount of particles passing through a sieve of $200 \mu \mathrm{m}$ (normal size of aperture: $150 \mu \mathrm{m}$ ) under manual stirring condition (60 rpm with a spatula) (IDF, 2013). In a real testing situation, however, it is not easy to maintain the
Table 2. Particle diameters of commercial skim milk powders with different heating strengths

\begin{tabular}{ccccc}
\hline \hline \multirow{2}{*}{ Skim milk powder } & \multicolumn{4}{c}{ Diameter $(\mu \mathrm{m})$} \\
\cline { 2 - 5 } & $10 \%$ & $50 \%$ & $90 \%$ & Mean \\
\hline Low heat & $17 \pm 2^{\mathrm{b}}$ & $52 \pm 1^{\mathrm{b}}$ & $93 \pm 3^{\mathrm{b}}$ & $55 \pm 1^{\mathrm{b}}$ \\
Medium heat & $19 \pm 1^{\mathrm{a}}$ & $64 \pm 1^{\mathrm{a}}$ & $122 \pm 2^{\mathrm{a}}$ & $68 \pm 2^{\mathrm{a}}$ \\
High heat & $14 \pm 1^{\mathrm{c}}$ & $39 \pm 2^{\mathrm{c}}$ & $83 \pm 1^{\mathrm{c}}$ & $44 \pm 1^{\mathrm{c}}$ \\
\hline
\end{tabular}

Assays were performed in triplicate. Mean \pm SD values in the same column with different superscripts are significantly different $(p<$ $0.05)$.

speed of manual stirring and samples sometimes stick to the spatula, causing errors in the measurement.

In the new testing method, dispersibility of samples was determined at regular time intervals without agitation and the results are expressed as a dispersibility curve. As shown in Fig. 2, medium- and low-heat SMP reached complete dispersion in 10 and $20 \mathrm{~min}$, respectively. In the case of high-heat SMP, even after 30 min, only $76 \%$ dispersion was reached. Dispersibility of the SMPs displayed the same trend as wettability. It has been reported that dispersibility also increases with increasing particle size, and decreases with an increasing proportion of fine particles below $90 \mathrm{~mm}$ (Fang et al., 2008). The newly devised method is easy to perform and gives highly reproducible results. The dispersibility curve straight forwardly demonstrates the relative ease of sample dispersion in an aqueous medium.

\section{Particle size analysis and microstructure of skim milk powders}

Mean size distributions of SMPs are shown in Table 2. The diameter of $50 \%$ of the SMP particles $\left(d_{50}\right)$ ranged from 39 to $64 \mu \mathrm{m}$, which were close to the size reported for commercial SMPs $(53 \mu \mathrm{m})$ by Fitzpatrick et al. (2004). The $d_{10}, d_{50}$ and $d_{90}$ of SMPs showed significant differences depending on the heating strength and the particle size increased in the order of high-, low- and mediumheat SMPs.

The surface characteristics and heterogeneity SMPs were observed by SEM. Spherical compact particles were evident for all SMPs whilst the high-heat SMP particles tended to have a smoother surface than did other SMP particles. As evident from the particle size analysis, medium-heat SMP contained larger particles than did the high-heat SMP. The high-heat SMP showed greater uniformity than did the other samples and this result was consistent with the report of Gaiani et al. (2011) that sphericity of dairy particles decreased as the particle size increased. 


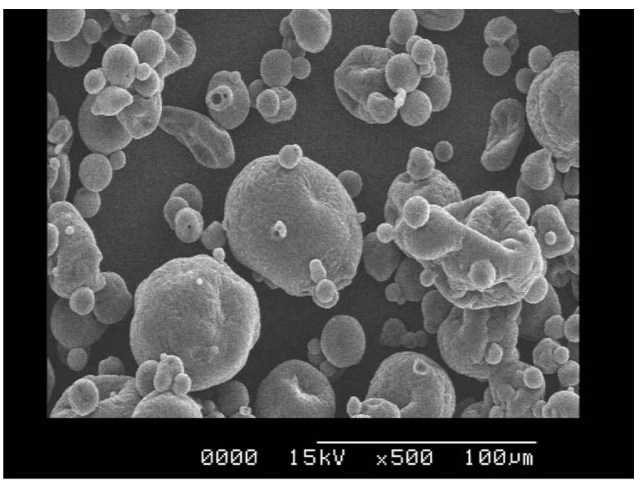

(a)

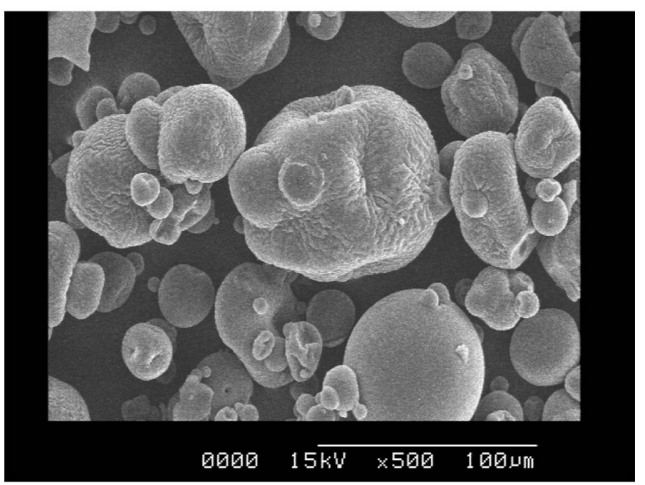

(b)

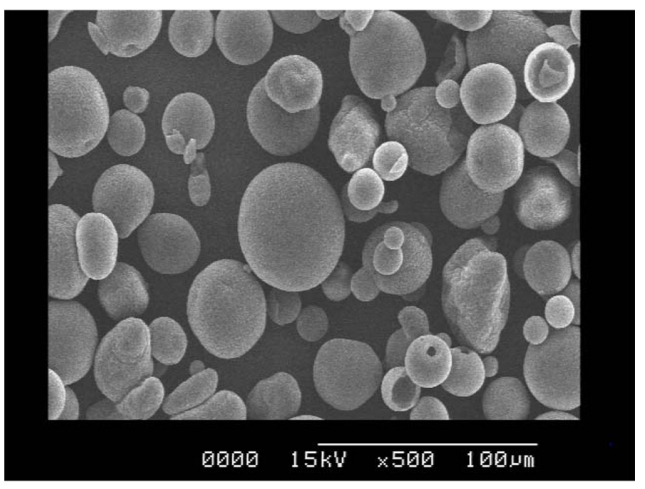

(c)

Fig. 3. Scanning electron microscopy images of commercial SMP with different heating strengths. (a) Low heatSMP, (b) Medium heat-SMP, (c) High heat-SMP. The samples were observed at $500 \times$ magnification.

\section{Conclusion}

SMPs produced at different heating strengths showed significant differences in wettability and dispersibility although their chemical compositions were quite similar. Wettability and dispersibility were correlated with the particle size of SMP. The high heat-SMP, which had the smallest particle size, showed the longest wetting time and a lower dispersibility than the low- and medium-heat
SMPs. Unlike existing methods, the new testing device allows for the determination of both the wettability and dispersibility of powders, and aids in the successful detection of differences among the samples.

\section{Acknowledgements}

This study was supported by High Value-added Food Technology Development Program funded by the Ministry of Agriculture, Food and Rural Affairs (MAFRA) (A2012-0447).

\section{References}

1. Davenel, A., Schuck, P., Mariette, F., and Brule, G. (2002) NMR relaxometry as a non-invasive tool to characterize milk powders. Lait 82, 465-473.

2. Fang, Y., Selomulya, C., and Chen, X. D. (2008) On measurement of food powder reconstitution properties. Drying Technol. 26, 3-14.

3. Fang, Y., Selomulya, C., Ainsworth, S., and Chen, X. D. (2011) On quantifying the dissolution behavior of milk protein concentrate. Food Hydrocoll. 25, 503-510.

4. Fitzpatrick, J. J., Iqbai, T., Delanry, C., Twomey, T., and Keogh, M. K. (2004) Effect of powder properties and storage conditions on the flowability of milk powders with different fat contents. J. Food Eng. 64, 435-444.

5. Gaiani, C., Banon, S., Scher, J., Schuck, P., and Hardy. J. (2005) Use of a turbidity sensor to characterize micellar casein powder rehydration: Influence of some technical effects. J. Dairy Sci. 88, 2700-2706.

6. Gaiani, C., Boyanova, P., Hussain, R., Pazos, M., Karam, M. C., Burgain, J., and Scher, J. (2011) Morphological descriptors and colour as a tool to better understand rehydration properties of dairy powders. Int. Dairy J. 21, 462-469.

7. Gaiani, C., Schuck, P., Scher, J., Desobry, S., and Banon. S. (2007) Dairy powder rehydration: Influence of protein state, incorporation mode, and agglomeration. J. Dairy Sci. 90, 570581.

8. Hla, P. K. and Hogekamp, S. (1999) Wetting behavior of instantized cocoa beverage powders. Int. J. Food Sci. Technol. 34, 335-342.

9. Hogekamp, S. and Schubert, H. (2003) Rehydration of food powders. Food Sci. Tech. Int. 9, 223-235.

10. IDF (2013) Instant dried milk - Determination of the dispersibility and wettability. IDF 087. Brussels, Belgium: International Dairy Foundation.

11. Kim, E. H. J., Chen, X. D., and Pearce, D. (2002) Surface characterization of four industrial spray-dried dairy powders in relation to chemical composition, structure and wetting property. Colloid Surface B 26, 197-212.

12. Kim, E. H. J., Chen, X. D., and Pearce, D. (2003). On the mechanism of surface formation and the surface compositions of industrial milk powders. Drying Technol. 21, 265-278. 
13. Mimouni, A., Deeth, H. C., Whittaker, A. K., Gidley, M. J., and Bhandari, B. R. (2009). Rehydration process of milk protein concentrate powder monitored by static light scattering. Food Hydrocoll. 23, 1958-1965.

14. Niro (2005) Wettability Niro method A 5 a. In GEA Niro
Analytical Methods. Soeborg, Denmark : GEA Niro.

15. Niro (2006) Wettability Niro method A 5 b. In GEA Niro Analytical Methods. Soeborg, Denmark : GEA Niro.

(Received 2014.10.21/Revised 2014.11.12/Accepted 2014.11.13) 